\title{
Early extubation in operating room after single-lung transplantation: a single institutional experience
}

\author{
Qing Ai", Hongsheng Deng", Danxia Huang", Qihua He, Guilin Peng, Weixiang Lu, Hengrui Liang, \\ Yunpeng Zhong, Jianfu Li, Shan Xiong, Bo Cheng, Caichen Li, Yi Zhao, Tuo Xing, Run Li, Ying Chen, \\ Wenhua Liang, Xin Xu, Jianxing He
}

Department of Thoracic Surgery and Oncology, the First Affiliated Hospital of Guangzhou Medical University, State Key Laboratory of Respiratory Disease, National Clinical Research Center for Respiratory Disease, Guangzhou Institute of Respiratory Health, Guangzhou, China Contributions: (I) Conception and design: W Liang, X Xu; (II) Administrative support: J He, X Xu; (III) Provision of study materials or patients: W Liang, X Xu; (IV) Collection and assembly of data: D Huang, Q Ai; (V) Data analysis and interpretation: D Huang, Q Ai, H Deng; (VI) Manuscript writing: All authors; (VII) Final approval of manuscript: All authors.

\#These authors contributed equally to this work.

Correspondence to: Xin Xu; Jianxing He. Department of Thoracic Surgery and Oncology, the First Affiliated Hospital of Guangzhou Medical University, State Key Laboratory of Respiratory Disease, National Clinical Research Center for Respiratory Disease, Guangzhou Institute of Respiratory Health, Guangzhou, China. Email: yichunrenjia@126.com; drjianxing.he@gmail.com.

Background Early endotracheal extubation in operating room (E-OR) after lung transplantation is rarely reported worldwide. Herein, we aim to explore the feasibility and safety of E-OR after lung transplantation and demonstrate its potential benefits.

Methods: This study is a single-center retrospective database analysis of 18 patients. All lung transplantation patients with E-OR attempted between June 2018 and September 2019 were included retrospectively. Perioperative variables, including ischemia time, total blood loss, blood lactic acid, the partial pressure of oxygen, partial pressure of oxygen/fraction of inspiration oxygen ratio, time of semi-open pulmonary artery occlusion clamp, extubation rate, and complications after E-OR, were analyzed. Data were compared using non-parametric tests and expressed as the median or number (percentage).

Results: Clinical data of 18 patients with E-OR attempted were collected. Overall, 15/18 (83.33\%) patients successfully underwent E-OR without reintubation. Reintubation occurred in 3/18 (16.67\%) patients; one patient presented with decreased blood oxygen saturation and unconsciousness, while two patients developed hypoxemia and respiratory failure after E-OR. Extracorporeal membrane oxygenation (ECMO) was not used postoperatively. No grade 3 primary graft dysfunction was observed and all eighteen patients were alive 1 year after the transplant. No postoperative hemodialysis and tracheotomy occurred. The median length of stay in the intensive care unit (ICU) for E-OR patients was 120 hours, the median length of postoperative hospital stay was 19 days, and the median hospitalization cost was 35,577 USD.

Conclusions: Early endotracheal extubation in operating room was feasible and did not delay postoperative recovery in these 18 lung transplantation recipients.

Keywords: Lung transplantation; early endotracheal extubation; anesthesia

Submitted Aug 12, 2020. Accepted for publication Feb 03, 2021.

doi: 10.21037/apm-20-1598

View this article at: http://dx.doi.org/10.21037/apm-20-1598

\section{Introduction}

Lung transplantation is an effective therapy for advancedstage lung diseases such as chronic obstructive pulmonary disease (COPD), idiopathic pulmonary fibrosis (IPF), $\alpha 1$ antitrypsin deficiency, and idiopathic pulmonary hypertension. More than 2,500 patients undergo lung 
transplants each year worldwide (1-3). The first singlelung transplantation was successfully performed in 1963 (4). Initially, lung transplantation patients must undergo mechanical ventilation in the intensive care unit (ICU), however, prolonged mechanical ventilation increases the risk of injury associated with bronchial anastomosis, which impairs postoperative rehabilitation. Moreover, immunocompromised lung transplant patients have a higher risk of nosocomial infections because of invasive mechanical ventilation and endotracheal intubation. Additionally, sedatives that are used in mechanical ventilation for inhibiting gastrointestinal movement can cause anesthesia-related complications (5). Thus the duration of tube placement is one of the important factors increasing the length and cost of hospitalization after lung transplantation $(6,7)$.

In the 1980s, early endotracheal extubation in the operating room (E-OR) was first applied in cardiac surgery, and later in liver transplantation $(8,9)$. E-OR can decrease the costs and the complications associated with mechanical ventilation such as hemodynamic instability, pulmonary colonization of gastrointestinal micro-organisms, sepsis, risks of barotrauma, and bronchial anastomosis leaks caused by the need for sedatives or opioids to inhibit gastrointestinal motility (10). The use of E-OR has been reported both in single-lung transplantation and bilateral transplantation in Europe and America. In 2003, Hansen et al. (10) retrospectively collected data of 53 patients extubated in the OR. Eleven patients needed reintubation within the first 24 hours because of respiratory insufficiency, pulmonary edema, hemorrhage, or pneumothorax. Similarly, in 2008, Augoustides et al. (11) compared the perioperative outcomes of E-OR to delayed tracheal extubation after lung transplantation and found that the major perioperative outcomes were equivalent. However, E-OR patients tended to have a higher rate of repeated tracheal intubation $(\mathrm{P}=0.09)$. Assenzo et al. (5) reported 35 patients extubated in the OR, with one requiring reintubation. Additionally, E-OR was associated with better $\mathrm{PaO}_{2} / \mathrm{FiO}_{2}$ ratio, more need for postoperative extracorporeal membrane oxygenation (ECMO), longer mechanical ventilation duration, a longer stay in the ICU, and lower Grade 3 primary graft dysfunction at $72 \mathrm{~h}$. However, data regarding the population receiving E-OR in Asia remains scarce. Moreover, the reasons for reintubation that mainly lead to the failure of E-OR, and the survival rate after E-OR should be given more attention.

This study aims to further assess the feasibility and safety of E-OR after lung transplantation, discussing the induction and maintenance of intraoperative anesthesia, and the criteria for E-OR. The economic and clinical benefits of E-OR for lung transplantation patients were also evaluated. This article is in accordance with the STROBE reporting checklist (available at http://dx.doi.org/10.21037/apm-201598).

\section{Methods}

The study protocol and methods were reviewed by the institutional ethics committee of the First Affiliated Hospital of Guangzhou Medical University (No. 2020-69). The data of all patients who underwent E-OR at the first affiliated hospital of Guangzhou Medical University between June 2018 to September 2019 were identified and consecutively collected retrospectively through electronic medical records. Informed consent of patients to have their data included for the study were signed in advance. The primary aim of this study was to evaluate the feasibility of E-OR. The study was conducted in accordance with the Declaration of Helsinki (as revised in 2013).

\section{Criteria for donors}

All donor lungs were obtained from Organ Procurement Organizations of Guangdong. The selection criteria of lung donors were: (I) age $<55$; (II) no history of extensive chest trauma; (III) duration of continuous mechanical ventilation $<5$ days; (IV) oxygenation index $>300 \mathrm{mmHg}(1 \mathrm{mmHg}$ $=0.133 \mathrm{kPa}$; (V) chest X-ray with relatively clear lung field; (VI) bronchoscopy of the airway was relatively clean; (VII) no obvious pathogenic bacteria were found in sputum culture.

\section{Inclusion and exclusion criteria for recipients}

Lung transplantation patients hospitalized in our center from June 2018 to September 2019 were included (Figure 1). The selection criteria were as follows. Inclusion criteria: (I) age is equal or greater than 18 years old; (II) consistent with lung transplant indications. Exclusion criteria: (I) patients with other serious medical diseases or complications; (II) patients who refuse to participate in the surgery.

\section{Anesthesia method}

The patient was placed in the left lateral position, with right $\mathrm{T}$ 6/7 paravertebral puncture and catheterization 


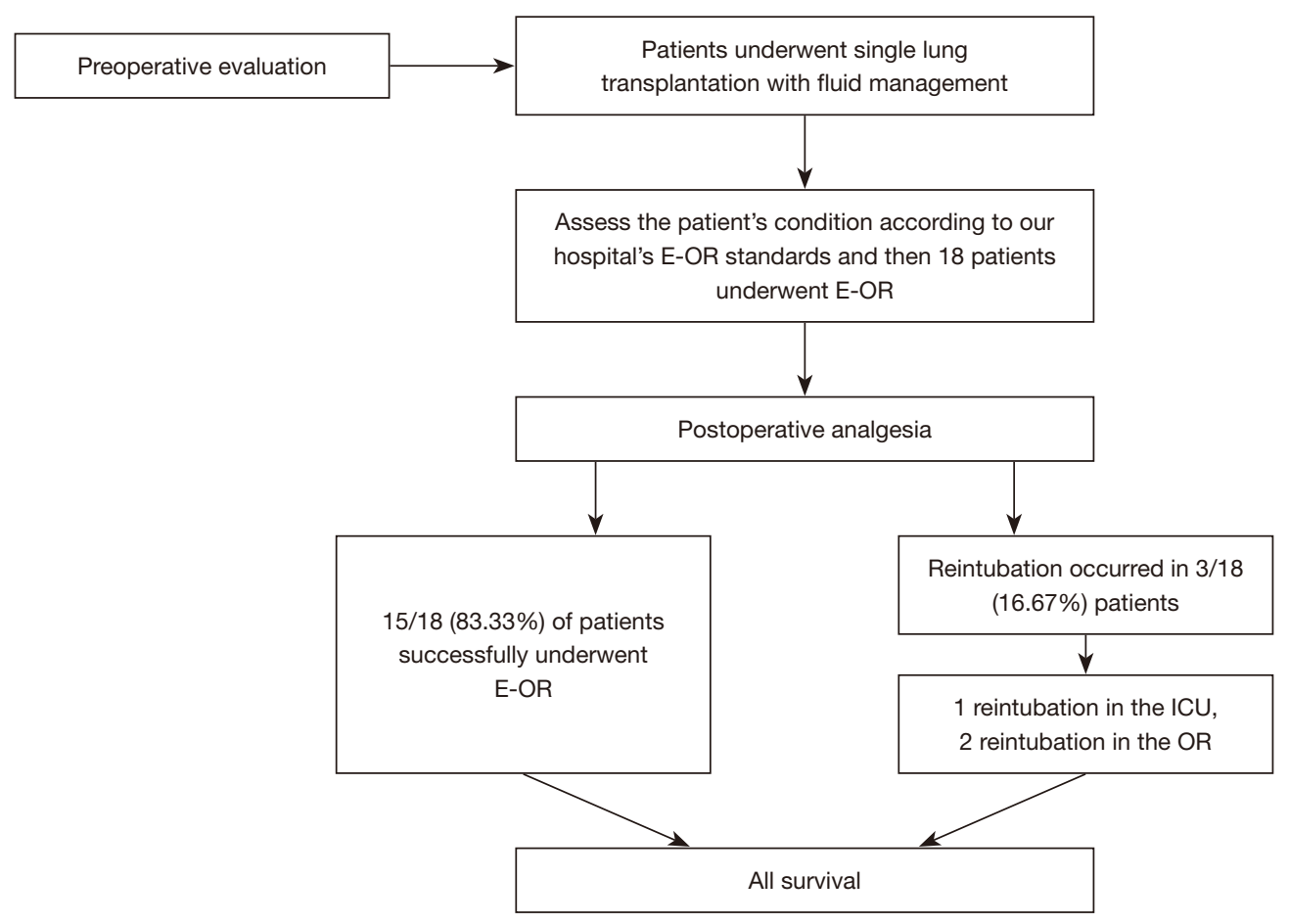

Figure 1 The procedures and outcomes of patients underwent E-OR. E-OR, early endotracheal extubation in the operating room.

for continuous paravertebral nerve block. Intraoperative anesthesia was maintained by intravenous pumping of propofol, dexmedetomidine, remifentanil, and cisatracurium, and by constant rate pumping of ropivacaine through a paravertebral indwelling tube. Routine noninvasive monitoring of heart rate (HR), blood pressure (BP), electrocardiograph (ECG), and oxygen saturation were performed after the patient entered the operating room. After the induction of anesthesia, a peripheral arterial catheter, deep venous catheter, and Swan-Ganz floating catheter were inserted. The radial artery catheter or brachial artery of the patient was connected to the detection catheter, which detected output, cardiac output index, stroke output, stroke output variation, extravascular pulmonary fluid index, and intrathoracic blood volume. Intraoperative continuous monitoring included: $\mathrm{HR}, \mathrm{BP}, \mathrm{SpO}_{2}, \mathrm{ECG}$, pulmonary artery pressure (PAP), central venous pressure, electroencephalogram (EEG), bispectral index, and bladder temperature.

\section{Surgical procedure}

A fourth intercostal posterolateral incision was made in the lateral chest wall. The donor lung was sequentially anastomosed with the main bronchus, pulmonary, and left atrial cuff of the recipient. When ventilation was resumed after anastomosis, special attention was paid to control tidal volume to avoid rapid and excessive expansion of transplanted lungs. No ECMO was performed during the operation, and the pulmonary artery occlusion forceps were partially opened for $10 \mathrm{~min}$ after anastomosis. Changes of intraoperative arterial blood gas and PAP and pulmonary graft reperfusion were observed. The surgical field was sufficiently hemostatic, a thoracic drainage tube was placed, and each layer of tissue was sutured layer by layer to complete the operation.

\section{Pre E-OR evaluation}

After 15 minutes of transplanted lung re-expansion, the condition of the patient was evaluated every 15 minutes. E-OR was applied when the patients were considered to meet the following criteria: (I) intraoperative urine volume over $30 \mathrm{~mL} / \mathrm{h}$, intraoperative blood loss less than $20 \%$ of normal circulating blood volume $(80 \mathrm{~mL} / \mathrm{kg})$; (II) Positive end-expiratory pressure reached $8 \mathrm{cmH}_{2} \mathrm{O}, \mathrm{PaCO}_{2}$ less than $60 \mathrm{mmHg}, \mathrm{SpO}_{2}$ greater than $90 \%$, oxygenation index (OI) greater than 150; (III) fiberoptic bronchoscopy examination 
showed that the bronchial anastomosis was smooth, patent, with only a small amount of bloody secretions; (IV) ECMO was not applied intraoperatively. Single-lung transplantation (SLTx) is a much less invasive procedure than double-lung transplantation (DLTx). SLTx can usually be performed without extracorporeal support. Therefore, we conclude that E-OR is more likely to occur in SLTx compared to DLTx, and we only performed E-OR in SLTx. From June 2018 to September 2019, a total of 64 patients underwent lung transplantation. Of those, 46 patients underwent SLTx, of which 18 patients met the criteria. E-OR decisions were made by the cardiothoracic surgeon and anesthesiologist after discussion.

\section{E-OR procedure}

The dosage of anesthetic drugs was gradually reduced (drugs include: cisatracurium, remifentanil, propofol, and dexmedetomidine, etc.). The measured value of the EEG dual-frequency index of the anesthesia depth monitor was $>70$. A rapid blood gas analyzer and a dual-level positive airway pressure noninvasive ventilator was prepared. Then patients were induced to resume spontaneous breathing after reasonable antagonistic drugs. When patients were in a calm state, after the recovery of spontaneous breathing, the endotracheal tube was replaced immediately with subsequent bilevel positive airway pressure non-invasive auxiliary ventilator assisted ventilation. Noninvasive ventilator parameters such as breath out at the end of the positive pressure (EPAP), inspiratory phase positive pressure (IPAP), $\mathrm{FiO}_{2}$ according to the patients' tidal volume adjustments, OI, and arterial blood gas were observed. When the patient used BiPAP noninvasive assisted ventilation, the following conditions were met: (I) BiPAP noninvasive assisted ventilation was smooth (II) no complaints of obvious pain and discomfort; (III) OI $>150 \mathrm{mmHg}$; (IV) $\mathrm{PaCO}_{2} \leq 50 \mathrm{mmHg}$ or lower than the preoperative level. BiPAP can be transferred to the ICU with a noninvasive auxiliary ventilator.

\section{Statistical analysis}

Continuous data are presented as the mean and standard deviation and were analyzed with two-sample Student's $t$-test for independent data. Categorical variables are given as a count and percentage and compared with the $\chi^{2}$ or Fisher exact test. All tests were 2-sided, with an a-level of 0.05 . SPSS software (SPSS version 25.0; IBM Corp, Armonk,
NY, USA) was used for all statistical evaluations.

\section{Results}

From June 2018 to September 2019, a total of 18 patients underwent E-OR. Of these patients, 15 (83.3\%) were successfully extubated in the OR without reintubation.

A total of 18 patients were eligible for this study, of which males comprised more than females $(83.3 \%$ vs. $16.7 \%)$. The median age of patients was 62 years old. Basic patient information: height, weight, and $\mathrm{BMI}$ are listed in Table 1. Overall, 10 of the 18 patients (55.6\%) were diagnosed with pulmonary hypertension preoperatively. Of the 18 patients who underwent preoperative plasmapheresis, only one patient had a history of thoracic surgery: a previous history of pulmonary tuberculosis and right upper pulmonary nodule resection ten years ago. Two patients had diabetes. Before lung transplantation, blood glucose level was controlled in all patients. No patients had undergone a preoperative tracheotomy. Lung transplantation patients had to wait in line for a donor lung. The median waiting time for lung transplantation was 34 days.

The median age of donors was 37.5 years old, and the median weight was $65[60-70] \mathrm{kg}$. All the organ donors had chest $\mathrm{X}$-rays taken. In the $\mathrm{X}$-ray results, only one case showed a small amount of secretion, one case was missed, and 16 cases were normal. The most common blood type was type $\mathrm{O}(77.8 \%)$, three donors were type A, and only one donor was type B. In the previous 6 months, 14 donors $(77.8 \%)$ had no history of smoking, whereas the smoking history of four donors was unknown. Another key point is the fiberoptic bronchoscopy results of the donors, 11 (61.1\%) fiberoptic bronchoscopy results were normal, $3(16.7 \%)$ showed dilute phlegm, the rest of the results were unknown. The median OI was 470 (411-511.75) and pulmonary vascular occlusion time was 229.5 (175.5-427.0) $\min$ (Table 1).

All 18 patients successfully received lung transplantations. Of all the patients, seven $(38.89 \%)$ patients underwent epidural catheter analgesia. The median operating time was 195.50 [185-249] min, the total blood loss was $200 \mathrm{~mL}$, blood lactate was $0.895 \mathrm{mmol} / \mathrm{L}$. Intraoperative outcomes of time, ischemia time, total blood loss, blood lactic acid, $\mathrm{PO}_{2}, \mathrm{PaO}_{2} / \mathrm{FiO}_{2}$ ratio, and time of semi-open pulmonary artery occlusion clamp are shown in Table 2. No patient underwent cardiopulmonary bypass and intraoperative ECMO. No major anesthesia-induced complications were observed after the transplantation. 
Table 1 Recipient and donor characteristics

\begin{tabular}{|c|c|}
\hline Characteristics & Value \\
\hline \multicolumn{2}{|l|}{ Recipient } \\
\hline Age (years) & 62 \\
\hline Gender (male-female) & $15-3(83.3-16.7)$ \\
\hline Height (cm) & $167.5(159.5-173)$ \\
\hline Weight (kg) & $59.5(50-66.6)$ \\
\hline BMI $\left(\mathrm{kg} / \mathrm{m}^{2}\right)$ & $21.15(18.04-23.08)$ \\
\hline \multicolumn{2}{|l|}{ Reason for lung transplantation [n (\%)] } \\
\hline $\begin{array}{l}\text { Chronic obstructive pulmonary } \\
\text { disease (COPD) }\end{array}$ & $8(44.5)$ \\
\hline Interstitial lung disease (ILD) & $8(44.5)$ \\
\hline Lymphangioleiomyomatosis & $1(5.5)$ \\
\hline Silicosis & $1(5.5)$ \\
\hline Pulmonary artery hypertension [n (\%)] & $10(55.6)$ \\
\hline Preoperative plasmapheresis [n (\%)] & 0 \\
\hline History of thoracic surgery [n (\%)] & $1(5.6)$ \\
\hline Diabetes [n (\%)] & $2(11.1)$ \\
\hline \multicolumn{2}{|l|}{ Donor } \\
\hline Age (years) & $37.5[14-48]$ \\
\hline Gender (male-female) & $15-3(83.3-16.7)$ \\
\hline Weight (kg) & $65[60-70]$ \\
\hline \multicolumn{2}{|l|}{ Smoking history [n (\%)] } \\
\hline No & $14(77.8)$ \\
\hline Unknown & $4(22.2)$ \\
\hline \multicolumn{2}{|l|}{ Blood type [n (\%)] } \\
\hline A & $3(16.7)$ \\
\hline B & $1(5.56)$ \\
\hline$A B$ & $0(0)$ \\
\hline $\mathrm{O}$ & $14(77.8)$ \\
\hline Secretion $[n(\%)]$ & $3(16.7)$ \\
\hline Oxygenation index (OI) & $470(411-511.75)$ \\
\hline $\begin{array}{l}\text { High emergency lung transplantation } \\
\text { [n (\%)] }\end{array}$ & 0 \\
\hline
\end{tabular}

Data are expressed as numbers (percentages), medians [25th75th percentiles], or medians (minimum-maximum). OI, oxygen and index.
Table 2 Intraoperative outcomes of lung transplantation patients who underwent E-OR

\begin{tabular}{lc}
\hline Variables & Value \\
\hline Operating time (minutes) & $195.50[185-249]$ \\
Intraoperative blood loss $(\mathrm{mL})$ & $200[100-450]$ \\
Blood lactate (mmol/L) & $0.895(0.725-1.23)$ \\
Semi-open pulmonary artery occlusion & $5[5-5]$ \\
forceps time (min) & \\
Ischemic time (min) & $229.5(175.5-427)$ \\
PaO & $167.5(148.8-202)$ \\
PaO & /FiO ${ }_{2}$ ratio \\
Thoracic epidural analgesia [n (\%)] & $268.5(222.5-297.0)$ \\
Anaesthetic induction complications & $7 / 18(38.89)$ \\
Severe hypotension & 0 \\
Difficult tracheal intubation & 0 \\
Pneumothorax & 0 \\
Cardiopulmonary bypass & 0 \\
Preoperative plasmapheresis [n (\%)] & 0 \\
Intraoperative ECMO & 0 \\
\hline
\end{tabular}

Data are expressed as numbers (percentages), medians [25th-75th percentiles], or medians (minimum-maximum) for age. $\mathrm{PaO}_{2}$, partial pressure of oxygen; $\mathrm{FIO}_{2}$, fraction of inspired oxygen; ECMO, extracorporeal membrane oxygenation.

Overall, 15 of the 18 patients underwent E-OR, with a success rate of $83.33 \%$. After E-OR, a patient presented with decreased blood oxygen saturation, increased HR, serous foam sputum, and blurred consciousness. This patient underwent nasal endotracheal intubation under endoscope in the ICU. After the intubation, acid correction, active fluid rehydration, colloid supplementation, and other supportive treatments were given. Considering the possibility of pulmonary ischemia-reperfusion injury in the patient, steroid pulse therapy was given. High-dose proglobulin was also given to suppress immunity, and albumin to alleviate pulmonary edema. The mechanical ventilation time was 240 minutes. Two patients developed hypoxemia and respiratory failure after extubation due to respiratory muscle weakness. The mechanical ventilation times were 509 and 240 minutes, respectively. No postoperative Grade-3 primary graft dysfunction or postoperative atrial fibrillation was observed. No postoperative ECMO was used, and no postoperative hemodialysis and tracheotomy occurred. The 
Table 3 Postoperative outcomes of lung transplantation patients who underwent E-OR

\begin{tabular}{|c|c|}
\hline Variables & Value \\
\hline Serum creatinine & 85.17 \\
\hline Arterial $\mathrm{pH}$ & 7.34 \\
\hline Arterial $\mathrm{pO}_{2}$ & 163.76 \\
\hline FEV1/FVC (\%) (range) & $64.22(39.03-99.40)$ \\
\hline $\begin{array}{l}\text { Grade } 3 \text { graft dysfunction } \\
\text { within } 72 \text { h [n (\%)] }\end{array}$ & $0(0)$ \\
\hline \multicolumn{2}{|l|}{$\operatorname{ECMO}(n)$} \\
\hline Reintubation [n (\%)] & $3(16.67)$ \\
\hline In OR & $1(5.56)$ \\
\hline In ICU & $2(11.11)$ \\
\hline \multicolumn{2}{|l|}{ Chest X-ray with $24 \mathrm{~h}$} \\
\hline Normal & 14 \\
\hline Atelectasis & 4 \\
\hline $\begin{array}{l}\text { Length of stay in the ICU } \\
\text { (hours) }\end{array}$ & $120(106.5-156.0)$ \\
\hline Length of hospital stay (days) & $19.5[16-28]$ \\
\hline Total costs (dollars) & $35,577.6(33,917.8-43,213.3)$ \\
\hline 1 month survival & $18[100]$ \\
\hline
\end{tabular}

Postoperative course. Data are expressed as numbers (percentages), medians [25th-75th percentiles], or medians (minimum-maximum) for age. FEV1, forced expiratory volume in the first second; FVC, forced vital capacity; ECMO, extracorporeal membrane oxygenation; $\mathrm{FIO}_{2}$, fraction of inspired oxygen; $\mathrm{PaO}_{2}$, partial pressure of oxygen; ICU, intensive care unit.

median duration of ICU stay was 120 hours and the median length of hospital stay was 19 days. The total cost includes all medical expenses incurred by the patient during this hospitalization, including preoperative examination, surgical expenses, and other hospitalization expenses, the cost of follow-up treatment of the patient after discharge from the hospital was excluded. The total cost of hospitalization was 35,577.6 dollars (Table 3).

\section{Discussion}

This study showed that E-OR after lung transplantation is feasible in select patients. Length of ICU stay, length of hospital stays, hospitalization cost, and intubation-related complications were less due to the application of E-OR. In addition, reintubation in the ICU or OR did not delay postoperative recovery.

Mechanical ventilation is a transitional treatment for respiratory management after lung transplantation. However, postoperative delays in ventilation may have many potential disadvantages, including bronchial injury and lung trauma, increased right ventricular workload, increased cardiac output, less renal perfusion, and increased stress and discomfort. Ventilator-associated pneumonia is also associated with increased mortality after lung transplantation (12-14).

Accelerated rehabilitation surgery refers to the comprehensive application of multidisciplinary techniques in the perioperative period, and the adoption of a series of systematic and targeted optimization measures that contribute to accelerated postoperative recovery, reduce the risk of postoperative complications and shortens hospital stay $(15,16)$. In terms of lung transplantation, an accelerated rehabilitation strategy comprise of optimizing anesthesia, reducing surgical trauma, shortening ICU stay, and early postoperative recovery. Early removal of the endotracheal tube in the operating room after lung transplantation is a specific measure to accelerate the application of rehabilitation surgery.

Reports of experiences with early extubation have been published in the lung transplantation field since 1999. Hansen et al. were the first to report E-OR after single-lung transplantation (10). Overall, 11/53 extubated patients required reintubation within the first $24 \mathrm{~h}$ due to respiratory insufficiency, pulmonary edema, hemorrhage, or pneumothorax. In 2003, Rocca et al. (17) concluded that early extubation is of feasibility in a retrospective study with 12 patients, 5/12 extubated patients experienced postoperative respiratory complications. In 2008, Augoustides et al. (11) evaluated the safety and success of early tracheal extubation in single-lung transplantation for COPD patients and concluded that this approach was safe and resulted in equivalent perioperative outcomes. In 2016, Felten et al. (18) reported a study where 41 patients underwent E-OR and 48 patients received traditional delayed extubation and found that postoperative complications occurred less frequently in the E-OR group, and a lower rate of Grade-3 primary graft dysfunction (0 vs. 19 patients, $\mathrm{P}<0.0001)$ and shorter $\operatorname{ICU}(5.0$, 3.7-7.2, vs. 11.5, 7.0-15.5, days) and hospital stays (22.0, 18.0-25.5, vs. 33.0, 25.0-56.5, days) $(\mathrm{P}<0.0001$ for both). All reports regarding E-OR believe that it is safe and feasible to perform E-OR after lung transplantation for qualified patients compared with the traditional delayed extubation 
technique. In this study, the length of hospital stays (5.0, 4.4-6.5, vs. 11.5, 7.0-15.5 days) and ICU stay (19.5, 16-28, vs. 33.0, 25.0-56.5 days) of E-OR patients were also shorter than in the traditional delayed extubation group of patients, which allows patients to pay less.

Pulmonary arterial hypertension, donor lungs that are given $e x$ vivo lung perfusion (EVLP), intraoperative $\mathrm{ECMO}$, and intraoperative hemodynamic instability all reduce the success rate of E-OR. In 2016, Felten et al. (18) proposed the indication of E-OR: (I) patients gradually wake up when propofol, fentanyl, and muscle relaxants are not used; (II) temperature $>36^{\circ} \mathrm{C}$; (III) intraoperative blood loss $<100 \mathrm{~mL} / \mathrm{h}$ and the hemodynamic indexes are stable; (IV) fiber bronchoscope shows no obvious edema; (V) OI $>200 \mathrm{mmHg}, \mathrm{PaCO}_{2}<50 \mathrm{mmHg}$, blood lactic acid $<3 \mathrm{mmol} / \mathrm{L}$; (VI) chest X-ray shows no abnormality (18). E-OR may be attempted in patients who meet the above conditions. Our center has a looser standard of $\mathrm{PaCO}_{2}$, with no more than $60 \mathrm{mmHg}$ compared to $50 \mathrm{mmHg}$ in Felten $e t a l$. This change allows more patients to undergo $\mathrm{E}-\mathrm{OR}$, and the success rate $(84.33 \%)$ is still acceptable. For patients with critical preoperative conditions, salvaging lung transplantation, the selection of transplant recipients should be very careful and adequate preoperative evaluation and preparation should be made.

Some perioperative outcomes have proven to be a prognostic factor of E-OR (19). Blood loss before E-OR, hemodynamics, oxygen partial pressure, carbon dioxide partial pressure, oxygen saturation, bronchoscopy results are all indicators that can objectively reflect the physiological state of ventilation, which also determines the time that the tube is drawn. The chest X-ray is an important evaluation standard to observe the secretions and lung recruitment situation after transplantation. In this study, chest X-ray 2 hours after the surgery showed that four patients have different levels of pulmonary atelectasis, these four patients were under intensive care.

Fluid management is also the key point to establish lung function and reduce ischemia-reperfusion injury in the early stage of lung transplantation (20). Monitoring the pulmonary fluid index and the blood volume index inside the chest cavity can better assess the pulmonary water and cardiac preload, which provide an important basis for E-OR (17). All 18 patients in this study received dynamic monitoring and timely adjustment of the above indicators, to obtain an opportunity for E-OR.

Since lung transplantation is a relatively traumatic operation, continuous and effective postoperative analgesia is an effective method to reduce oxygen consumption, reduce ventilator support, and facilitate the excretion of postoperative cough and sputum, which is good for postoperative recovery (21). In this study, seven patients underwent preoperative ultrasound-guided epidural block. The analgesic effect was satisfied with the administration of ropivacaine during and after the surgery. For patients who plan to be extubated in the OR, continuous and adequate epidural block is an essential condition.

E-OR extubation did not lead to an increased postoperative risk in our series and may identify patients with more favorable outcomes. In this study, the success rate of $\mathrm{E}-\mathrm{OR}$ was $84.33 \%$. In a study done at another center, with 91 patients, $53 \%$ underwent E-OR and $21 \%$ of these patients were reintubated (10), which was similar to the outcome of this study. Notably, the three patients that underwent reintubation were not associated with severe complications, which indicates that timely reintubation in the OR/ICU could alleviate the patients' situation.

After extubation, the patient was observed for about $1 \mathrm{~h}$ to determine whether safe transport to the ICU was possible. Measures of evaluation included patients with good pain tolerance and no significant respiratory distress (arterial blood gas analysis: $\mathrm{OI}>200 \mathrm{mmHg}$ and $\mathrm{PaCO}_{2}$ $<50 \mathrm{mmHg}$ ). However, the patient should be actively reintubated in the case of (I) surgical complications, such as bleeding, anastomotic leakage, etc.; (II) the dosage of vasoactive drugs is increased for unstable circulation; (III) respiratory failure must be given greater respiratory support through endotracheal intubation; (IV) severe primary graft loss (Grade 3) results in total or major loss of transplanted lung function $(10,11)$.

All 18 patients received single-lung transplantations. The chest X-rays of the patients showed that the exudation of the transplanted lung decreased $48 \mathrm{~h}$ after surgery. The OI gradually increased after a short period of decline, and the fluid secretions of the transplanted lungs under bronchoscopy also gradually reduced $48 \mathrm{~h}$ after the surgery, which demonstrated that the postoperative recovery was ideal. There have been studies on E-OR in patients with double-lung transplantation or even patients supported by ECMO intraoperatively. The overall success rate of extubation is more than $90 \%$, and the recent survival rate of patients with early extubation is better than that of patients that have reintubation in ICU $(5,18)$. For lung transplant recipients, adequate preoperative examination and evaluation, continuous and effective epidural block, intraoperative vital signs, and monitoring of important 
indicators are important guarantees to improve the success rate of early extubation. By screening eligible patients and assessing perioperative outcomes and physical indicators, we concluded that E-OR after unilateral lung transplantation is feasible, and can reduce the length of ICU stays, total hospital stays, hospitalization costs, and save valuable medical resources. More practical and effective monitoring methods are needed to ensure E-OR after lung transplantation and promote the rapid recovery of lung transplantation patients.

This study made no direct comparison between E-OR and non-E-OR patients. However, when compared with ICU-extubation patients presented in the study by Assenzo et al. (5), the $\mathrm{PaO}_{2} / \mathrm{FiO}_{2}$ ratio of the E-OR group in the present study was better $(268.5,222.5-297.0, v s .206,144$ $357)$ and Grade 3 primary graft dysfunction at $72 \mathrm{~h}(0 \mathrm{vs}$. $10 \%, 24 \%$ ) was lower, which demonstrated the superiority of this strategy.

Limitations still exist in this study. First, the sample size was small, however, early extubation in lung transplantation patients is not a routine procedure in clinical practice. Second, the retrospective design might be associated with selection bias. Third, the follow-up time of all lung transplantation patients is insufficient. We only reported perioperative outcomes rather than long-term outcomes.

\section{Acknowledgments}

We thank International Science Editing (www. internationalscienceediting.com) for its linguistic assistance during the preparation of this manuscript.

Funding: None.

\section{Footnote}

Reporting Checklist: The authors have completed the STROBE reporting checklist. Available at http://dx.doi. org/10.21037/apm-20-1598

Data Sharing Statement: Available at http://dx.doi. org/10.21037/apm-20-1598

Conflicts of Interest: All authors have completed the ICMJE uniform disclosure form (available at http://dx.doi. org/10.21037/apm-20-1598). The authors have no conflicts of interest to declare.

Ethical Statement: The authors are accountable for all aspects of the work in ensuring that questions related to the accuracy or integrity of any part of the work are appropriately investigated and resolved. The study protocol and methods were reviewed by the institutional ethics committee of the First Affiliated Hospital of Guangzhou Medical University (No. 2020-69). Informed consent of patients to have their data included for the study were signed in advance. The study was conducted in accordance with the Declaration of Helsinki (as revised in 2013).

Open Access Statement: This is an Open Access article distributed in accordance with the Creative Commons Attribution-NonCommercial-NoDerivs 4.0 International License (CC BY-NC-ND 4.0), which permits the noncommercial replication and distribution of the article with the strict proviso that no changes or edits are made and the original work is properly cited (including links to both the formal publication through the relevant DOI and the license). See: https://creativecommons.org/licenses/by-nc-nd/4.0/.

\section{References}

1. Thabut G, Christie JD, Kremers WK, et al. Survival differences following lung transplantation among US transplant centers. JAMA 2010;304:53-60.

2. George PM, Patterson CM, Reed AK, et al. Lung transplantation for idiopathic pulmonary fibrosis. Lancet Respir Med 2019;7:271-82.

3. Fuehner T, Kuehn C, Hadem J, et al. Extracorporeal membrane oxygenation in awake patients as bridge to lung transplantation. Am J Respir Crit Care Med 2012;185:763-8.

4. Ettinger NA, Bailey TC, Trulock EP, et al. Cytomegalovirus infection and pneumonitis. Impact after isolated lung transplantation. Washington University Lung Transplant Group. Am Rev Respir Dis 1993;147:1017-23.

5. Assenzo V, Assenzo C, Filippo R, et al. The feasibility of extubation in the operating room after bilateral lung transplantation in adult emphysema patients: an observational retrospective study. Eur J Cardiothorac Surg 2018;54:1128-33.

6. Mandell MS, Lezotte D, Kam I, et al. Reduced use of intensive care after liver transplantation: influence of early extubation. Liver Transpl 2002;8:676-81.

7. Weill D. Access to Lung Transplantation. The Long and Short of It. Am J Respir Crit Care Med 2016;193:605-6.

8. Aneja S, Raina R. Immediate postoperative extubation after liver transplantation at our centre: A report of two 
cases. Indian J Anaesth 2011;55:392-4.

9. Winch PD, Staudt AM, Sebastian R, et al. Learning From Experience: Improving Early Tracheal Extubation Success After Congenital Cardiac Surgery. Pediatr Crit Care Med 2016;17:630-7.

10. Hansen LN, Ravn JB, Yndgaard S. Early extubation after single-lung transplantation: analysis of the first 106 cases. J Cardiothorac Vasc Anesth 2003;17:36-9.

11. Augoustides JG, Watcha SM, Pochettino A, et al. Early tracheal extubation in adults undergoing single-lung transplantation for chronic obstructive pulmonary disease: pilot evaluation of perioperative outcome. Interact Cardiovasc Thorac Surg 2008;7:755-8.

12. Feltracco P, Serra E, Barbieri S, et al. Noninvasive ventilation in postoperative care of lung transplant recipients. Transplant Proc 2009;41:1339-44.

13. Pigula FA, Gandhi SK, Ristich J, et al. Cardiopulmonary transplantation for congenital heart disease in the adult. J Heart Lung Transplant 2001;20:297-303.

14. Guay J, Ochroch EA, Kopp S. Intraoperative use of low volume ventilation to decrease postoperative mortality, mechanical ventilation, lengths of stay and lung injury in adults without acute lung injury. Cochrane Database Syst Rev 2018;7:CD011151.

15. Impact of Enhanced Recovery After Surgery and Fast Track Surgery Pathways on Healthcare-associated

Cite this article as: Ai Q, Deng H, Huang D, He Q, Peng G, Lu W, Liang H, Zhong Y, Li J, Xiong S, Cheng B, Li C, Zhao Y, Xing T, Li R, Chen Y, Liang W, Xu X, He J. Early extubation in operating room after single-lung transplantation: a single institutional experience. Ann Palliat Med 2021;10(4):4134-4142. doi: 10.21037/apm-20-1598
Infections. Results From a Systematic Review and Metaanalysis: Erratum. Ann Surg 2017;266:e123.

16. Shewale JB, Correa AM, Baker CM, et al. Impact of a Fast-track Esophagectomy Protocol on Esophageal Cancer Patient Outcomes and Hospital Charges. Ann Surg 2015;261:1114-23.

17. Rocca GD, Coccia C, Costa GM, et al. Is very early extubation after lung transplantation feasible? J Cardiothorac Vasc Anesth 2003;17:29-35.

18. Felten ML, Moyer JD, Dreyfus JF, et al. Immediate postoperative extubation in bilateral lung transplantation: predictive factors and outcomes. Br J Anaesth 2016;116:847-54.

19. Blaszczyk B, Wronska B, Klukowski M, et al. Factors Affecting Breathing Capacity and Early Tracheal Extubation After Liver Transplantation: Analysis of 506 Cases. Transplant Proc 2016;48:1692-6.

20. Geube MA, Perez-Protto SE, McGrath TL, et al. Increased Intraoperative Fluid Administration Is Associated with Severe Primary Graft Dysfunction After Lung Transplantation. Anesth Analg 2016;122:1081-8.

21. Pottecher J, Falcoz PE, Massard G, et al. Does thoracic epidural analgesia improve outcome after lung transplantation? Interact Cardiovasc Thorac Surg 2011;12:51-3. 